\title{
CORRECTION
}

\section{Correction to: Low-dose decitabine enhances the effect of PD- 1 blockade in colorectal cancer with microsatellite stability by re-modulating the tumor microenvironment}

Ganjun Yu, Yanfeng Wu, Wenying Wang, Jia Xu, Xiaoping Lv, Xuetao Cao and Tao Wan

Cellular \& Molecular Immunology (2020) 17:111-112; https://doi.org/10.1038/s41423-019-0340-z

Correction to: Cellular \& Molecular Immunology 2019 (16), 401-409, https://doi.org/10.1038/s41423-018-0026-y, published online 5 April 2018.

In the published version of this paper, we repeatedly copied the image of the DAC-7 day as the DAC-14 day. The corrected Fig. 6 is provided below. The figure legend was revised in order to avoid confusion and is shown below for reference.

Although we regret our mistake during figure assembly and would like to apologize for any inconvenience it may have caused, we did not manipulate our data in any way. This unintentional error also has no bearing on the work's scientific conclusions. 
A

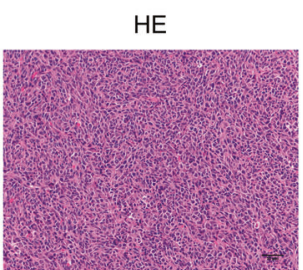

DAC-3 day

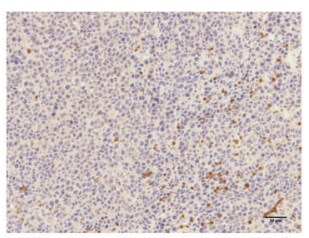

C
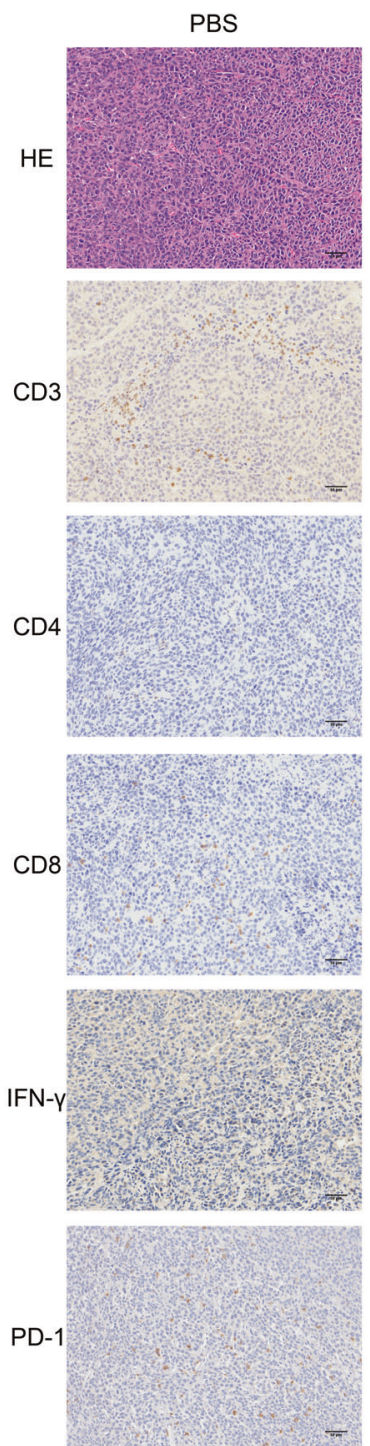

pre-DAC

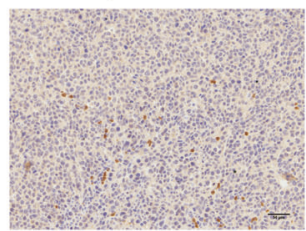

DAC-7 day

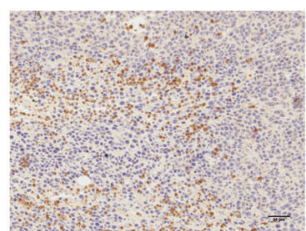

Cytidine
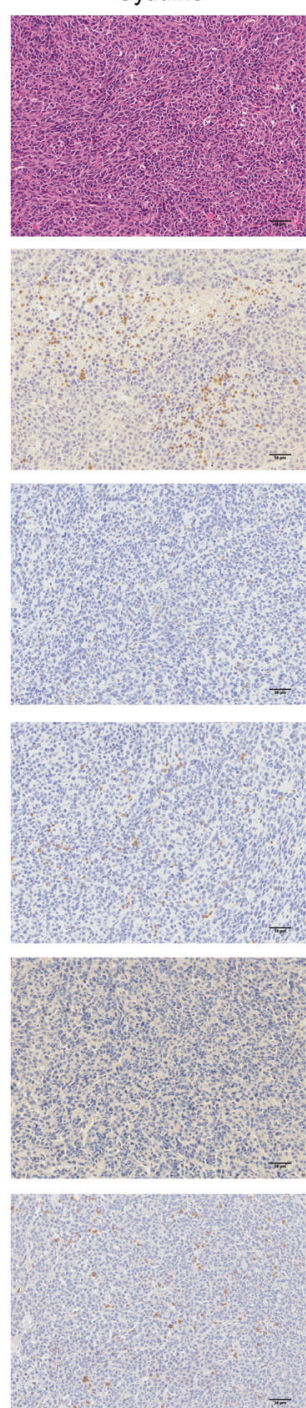

DAC-1 day

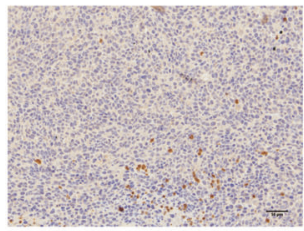

DAC-14 day

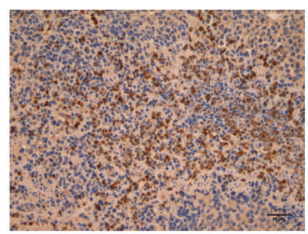

DAC
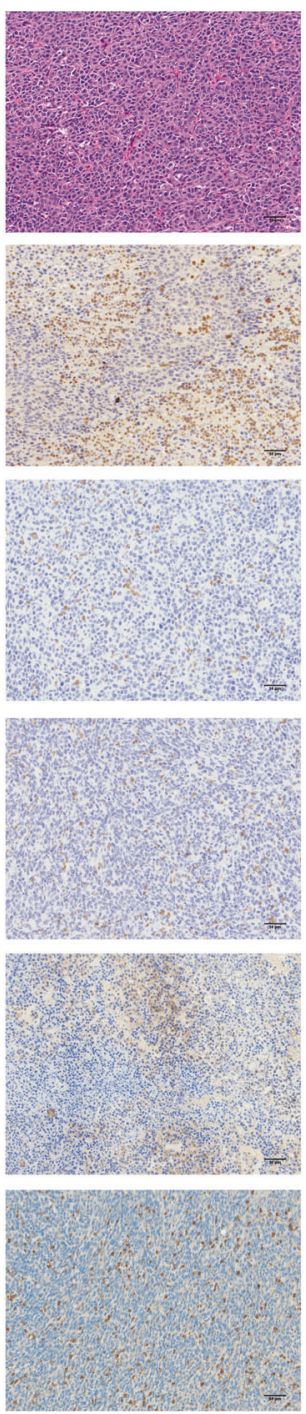

B

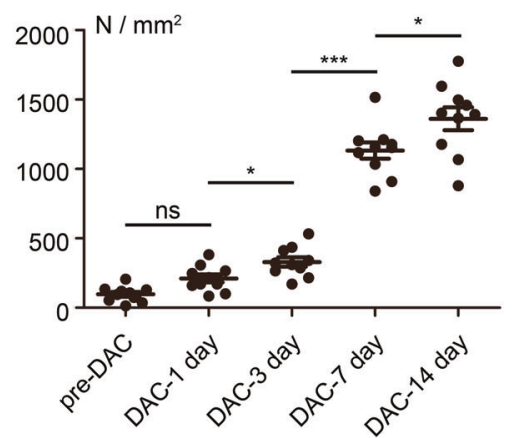

D
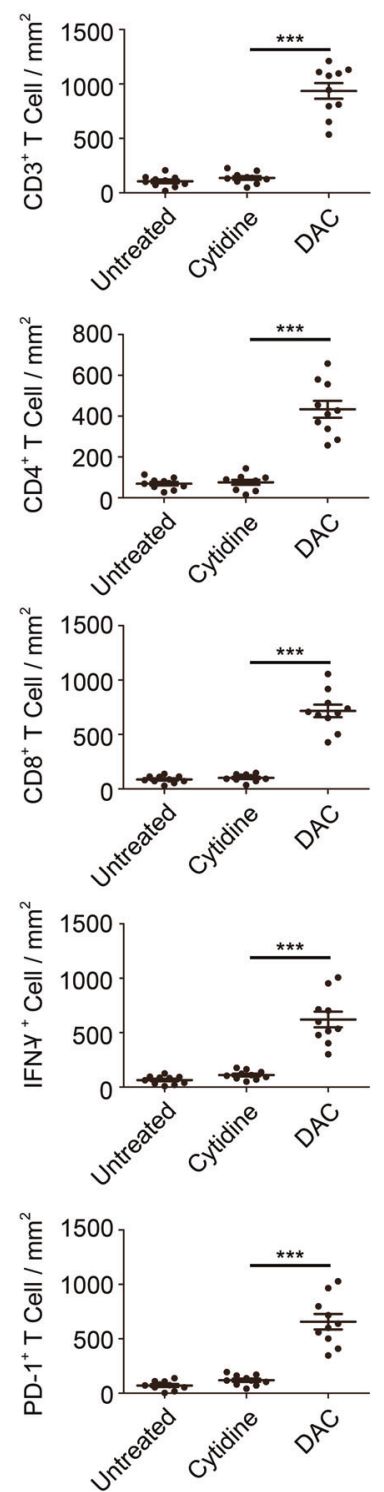

Fig. 6 Re-modulation of the tumor microenvironment by low-dose DAC in CT26-bearing mice. a The mice were inoculated with the tumor, and beginning 7 days later, each mouse received peritoneal injections of $20 \mu \mathrm{g}$ of DAC for 5 consecutive days. The last day of injection was recorded as DAC-0 day. The mice were sacrificed at DAC-1, DAC-3, DAC-7 and DAC-14 days. The tumor sections were stained for CD3 by IHC. $\mathbf{b}$ The stained slides were analyzed using the PerkinElmer Vectra 3 automated, high-throughput quantitative pathology imaging system. CD3-positive T cells were counted under $\times 200$ magnification. From each slide, ten fields were selected for analysis. The results were analyzed using ANOVA. ${ }^{*} p<0.05,{ }^{* * *} p<0.001, \mathrm{~ns}$ indicates no significant difference. c The tumors on DAC-7 day from the PBS-treated, cytidine-treated and DAC-treated mice were stained for CD3, CD4, CD8, IFN- $\gamma$ and PD-1. d The stained slides were analyzed using the PerkinElmer Vectra 3 automated, high-throughput quantitative pathology imaging system. The positive cells were counted under $\times 200$ magnification. From each slide, ten fields were selected for analysis. The results were analyzed using ANOVA. ${ }^{* * *} p<0.001$. 